\title{
A Robust Facial Feature Point Tracker using Graphical Models
}

\author{
Serhan Coşar, Müjdat Çetin, Aytül Erçil \\ Sabancı University \\ Faculty of Engineering and Natural Sciences \\ Orhanl1- Tuzla, 34956 İstanbul, TURKEY \\ serhancosar@su.sabanciuniv.edu, \{mcetin,aytulercil $\} @$ sabanciuniv.edu
}

\begin{abstract}
In recent years, facial feature point tracking becomes a research area that is used in human-computer interaction (HCI), facial expression analysis, etc. In this paper, a statistical method for facial feature point tracking is proposed. Feature point tracking is a challenging topic in scenarios involving arbitrary head movements and uncertain data because of noise and/or occlusions. As a natural human action, people move their heads or occlude their faces with their hands or fingers. With this motivation, a graphical model that uses temporal information about feature point movements as well as the spatial relationships between such points, which is updated in time to deal with different head pose variations, is built. Based on this model, an algorithm that achieves feature point tracking through a video observation sequence is implemented. Also, an occlusion detector is proposed to automatically detect occluded points. The proposed method is applied on $2 D$ gray scale video sequences consisting head movements and occlusions and the superiority of this approach over existing techniques is demonstrated.
\end{abstract}

\section{Introduction}

Facial feature point tracking is an important step in problems such as video based facial expression analysis and human-computer interaction. Generally, a facial expression analysis system consists of three components: feature detection, feature tracking, and expression recognition. Feature detection involves detecting some distinguishable points that can define the movement of facial components. This may involve, detection of eyes, eye brows, mouth or feature points of these components. Then comes the tracking part which consists of tracking the detected feature points. Finally, according to tracking results of these feature points, the expression recognition component outputs results such as happy, sad, surprised, etc. This paper is a preliminary work of facial feature point tracking. For simplicity, proposed work is applied for only eye feature points. The aim is to produce a robust tracker in the cases of arbitrary head movements and insufficient data because of occlusion and/or noise.

For feature point tracking, roughly there are two classes of methods in literature: general purpose approaches, face-specific approaches. One of the general purpose approaches is moving-point-correspondence methods $[1,2]$. The smooth motion, limited speed, and no occlusion assumptions make these methods inapplicable to facialfeature tracking. Another approach is the patch correlation method [3,4] which is sensitive to illumination and object-pose variations. There are also some optical-flow based methods [5, 6] which often assume image-intensity constancy for corresponding pixels, which may not be the case for facial features.

Compared with the general-purpose feature-tracking techniques, the face-specific methods are more effective. There are methods which use Gabor Filters $[7,8]$ to track facial feature points. Also, active appearance (AAM) or active shape models (ASM) $[9,10,11]$ are used to track feature points based on a face model. Additionally, the work in [7] tracks feature points based on spatial and temporal connections using non-parametric methods.

Generally, feature point tracking is done by using a temporal model that is based on pixel values. Consequently, these methods are sensitive to illumination and pose changes, and ignore the spatial relationships between feature points. This affects the tracking performance adversely, causes drifts and physically unreasonable results when the data are noisy or uncertain due to occlusions. In [12], a method where the spatial relationships are taken into account is proposed for contour tracking. However, since the method is based on non-parametric estimation techniques, it is rather computationally intensive. In addition, most of the recent methods works for videos when there is no occlusion on face which is not a case for real human actions. Not dealing with occlusion results lossy feature point tracking. Another disadvantage of recent works is the limitation of possible head movements which is again not a case for real human actions and causes drifts when head moves more than assigned limits. 
In this paper feature point tracking is performed in a framework that incorporates the temporal and spatial information between feature points. This framework is based on graphical models that have recently been used in many computer vision problems. The model is based on a parametric model in which the probability densities involved are Gaussian. The parametric nature of the models makes the method computationally efficient. The spatial connections between points allow the tracking to continue reasonably well by exploiting the information from neighboring points, even if a point disappears from the scene or cannot be observed. Another advantage of the spatial connections is to build a whole model by binding the feature points and prevent the possible drifts occurring because of head movements. The feature values from video sequences are based on Gabor filters. Filters are used in a way to detect the edge information in the image, to be sensitive to different poses, orientations and different feature sizes. Also, an occlusion detector based on the Gabor filter outputs is proposed to automatically detect occluded points. Tests on videos containing head movements and occlusions showed that tracking of facial feature points is performed successfully.

\section{Proposed Method}

\subsection{Preprocessing \& Occlusion Detection}

Gabor filters are used as a preprocessing stage for the observation section of the proposed work. The filters are selected as in [13]. Then as in [7], frames are convolved with 24 filters consisting of 6 different orientations and 4 different wavelengths. The magnitude and phase of the complex outputs of the filtering for the first frame and next frames is compared using the similarity metric in [13]. This produces similarity values for every point in the convolution region. The location of the best match, with the highest similarity value, is used as the observation data for the feature point in the next frame. Since feature extraction is not in the scope of this paper, feature points are marked for the first frame.

The output value of the similarity metric gives a quantitative information about how much these points are similar. For example, the similarity value for an occluded point will be low, but the similarity value for an unoccluded point will be high, as illustrated in Figure 1. By thresholding similarity values, occlusion can be detected for any point in any frame. A plot of these values for the video sequences that the proposed method is applied on is given in section 3.

\subsection{Graphical Models}

Graphical models can be defined as a marriage of graph theory and probability theory. The visualization property of graph theory makes even a complex model clear and understandable. This provides a powerful, general framework for developing statistical models of computer vision problems.

Generally a graph $G$ is defined by a set of nodes $V$, and a corresponding set of edges $E$. The neighborhood of a node $s \in V$ is defined as $N(s)=\{t \mid(s, t) \in E\}$. The models are divided into two main categories: directed and undirected graphs. Directed graphs are graphs in which there is a causal relation between random variables. In undirected graphs the relation is bidirectional.

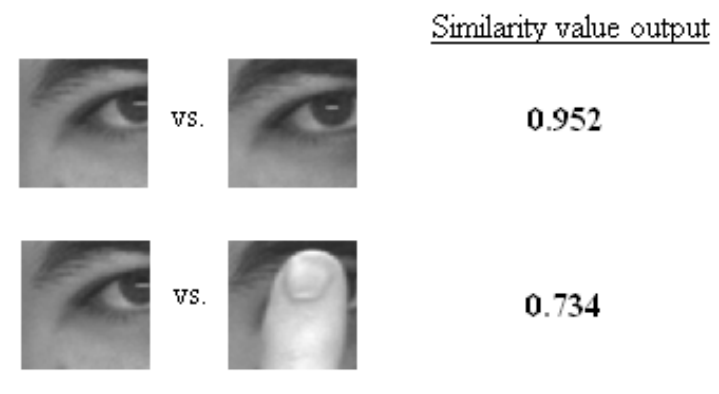

Figure 1. Similarity value outputs

Graphical models usually associate each node $s \in V$ with an unobserved, hidden random variable $\left(x_{s}\right)$, and a noisy local observation $\left(y_{s}\right)$. Let $x=\left\{x_{s} \mid s \in V\right\}$ and $y=\left\{y_{s} \mid s \in V\right\}$ denote the sets of all hidden and observed variables, respectively. This simply makes the factorization of a joint probability function $p(x, y)$ as shown below.

$$
p(x, y)=\frac{1}{Z} \prod_{(s, t) \in E} \psi_{s, t}\left(x_{s}, x_{t}\right) \prod_{(s) \in V} \psi_{s}\left(x_{s}, y_{s}\right)
$$

Here, $\psi_{s, t}\left(x_{s}, x_{t}\right)$ is the edge potential between hidden variables. The other term, $\psi_{s}\left(x_{s}, y_{s}\right)$ is the observation potential.

The graphical model that is used in the proposed method, when we track two feature points, is shown in Figure 2. Each hidden variable $\left(x_{s}\right)$ in the model is a vector with four elements. Assuming the movement of the feature points are in 2D, these four elements are $\mathrm{x}$-coordinates, $\mathrm{y}$-coordinates, velocity at $\mathrm{x}$-axis and velocity at $\mathrm{y}$-axis of the points. The observed nodes $\left(y_{s}\right)$ are a vector with two elements; $\mathrm{x}$ -coordinates and y-coordinates of observation data.

Actually, the feature points are 3D points that move in 3D real-world coordinate system. But, building a model in $3 \mathrm{D}$ is a hard problem, because monocular 2D images doesn't give enough information on z-axis movements. There are two main methods for going from 2D coordinate system to 3D coordinate system; stereo vision and motion. Stereo vision isn't in the scope of this paper. According to 
some trials, using motion to get the insufficient information on z-axis doesn't give stable results. So, the model is built in 2D. To make the model sufficient to movements in 3D, some methods are explained in section 2.4.

In this notation, $\left(x_{t}^{1}\right)$ means hidden variable of the first feature point at time $\mathrm{t}$ and $\left(y_{t+1}^{2}\right)$ is the observed variable of the second feature point at time $t+1$.

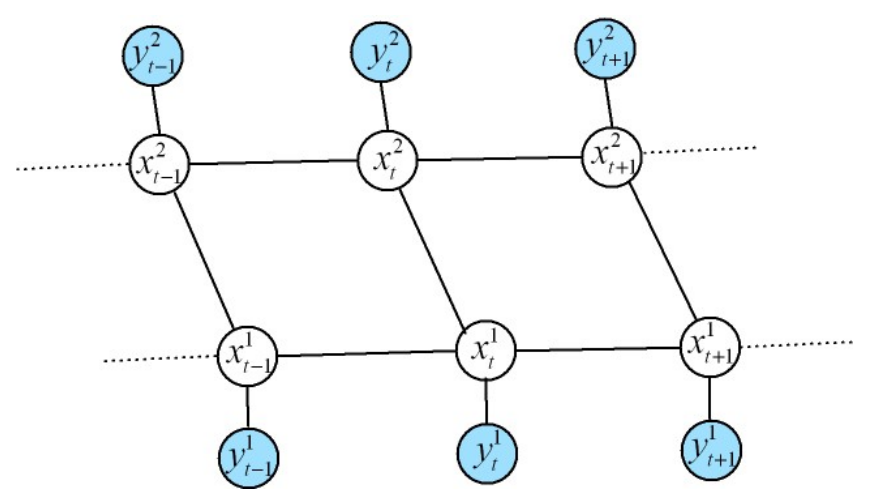

Figure 2. The graphical model used in this work

The selection of the edge potentials mentioned in this section is explained in 2.3-2.5.

\subsection{Temporal Model}

The temporal model makes the connection of the feature points with the previous value of the points. This is based on the translation model shown below.

$$
x_{t+1}=A \cdot x_{t}+w \quad w \backsim N(0, Q)
$$

Here, $A$ is the translation matrix. $Q$ is the covariance matrix of the noise which is a normal distribution with zeromean. Assuming the points move with a constant velocity and the point coordinates and velocities are independent of each other, these are selected as:

$$
A=\left(\begin{array}{cccc}
1 & 0 & 1 & 0 \\
0 & 1 & 0 & 1 \\
0 & 0 & 1 & 0 \\
0 & 0 & 0 & 1
\end{array}\right) Q=\left(\begin{array}{cccc}
\sigma_{x}^{2} & 0 & 0 & 0 \\
0 & \sigma_{y}^{2} & 0 & 0 \\
0 & 0 & \sigma_{u}^{2} & 0 \\
0 & 0 & 0 & \sigma_{v}^{2}
\end{array}\right)
$$

Hence the temporal connection between two nodes involves a Gaussian distribution and the edge potential can be defined as follows:

$\psi_{t, t+1}\left(x_{t}, x_{t+1}\right)=$
$\alpha \exp \left\{-\frac{1}{2}\left[\begin{array}{ll}x_{t+1}^{T} & x_{t}^{T}\end{array}\right]\left[\begin{array}{cc}Q^{-1} & -Q^{-1} A \\ -A^{T} Q^{-1} & A^{T} Q^{-1} A\end{array}\right]\left[\begin{array}{c}x_{t+1}^{T} \\ x_{t}^{T}\end{array}\right]\right\}$

\subsection{Spatial Model}

The spatial model defines the spatial connection between feature points. This connection is selected to simply use the expected spatial distance between feature points, for example; the distance between the eye corners. According to these, the spatial connection is selected as below:

$$
\begin{array}{r}
\psi_{1,2}\left(x_{1}, x_{2}\right)=\alpha \exp \\
\left\{\left[x_{1}-\left(x_{2}-\triangle x\right)\right]^{T} \Sigma^{-1}\left[x_{1}-\left(x_{2}-\triangle x\right)\right]\right\}
\end{array}
$$

Here, $\triangle x$ is a four elements vector containing the distance on $\mathrm{x}$-axis and $\mathrm{y}$-axis as: $\triangle x=\left[\begin{array}{lllll}\triangle x_{x} & \triangle x_{y} & 0 & 0\end{array}\right]^{T}$. Because the distance will change, the challenging thing, as explained in section 2.2 , is to find the expected distance between feature points in the cases of head pose variations like; rotation, z-translation. But, the proposed spatial model is a general model for the point movements in 3D. The spatial distances between points can be updated in two ways: One is by using the pose output of a head pose tracker algorithm that runs in parallel. Another way is to find the same information by using reliably tracked feature points. This method is explained below.

As illustrated in Figure 3, the ratio of the distances $(|A B|,|B C|)$ between three points $(A, B, C)$ will be same even if these points move to any arbitrary position $\left(A^{\prime}, B^{\prime}, C^{\prime}\right)$. This theorem is called affine-ratio. Affine-

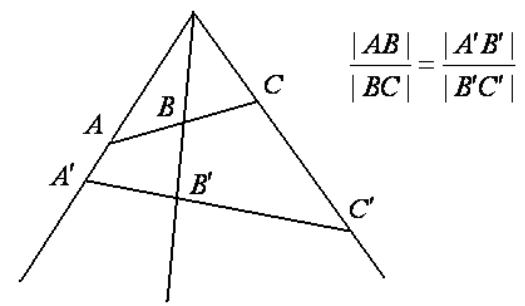

Figure 3. Affine-Ratio

ratio can be used for the distances on $\mathrm{x}$-axis and $\mathrm{y}$-axis between feature points. By using the spatial distances on $\mathrm{x}$ and $\mathrm{y}$ axes between reliably tracked points, the spatial distances on $\mathrm{x}$ and $\mathrm{y}$ axes for the other lossy tracked points can be found by affine-ratio, as illustrated in Figure 4. This method makes the spatial model updated in time and gives an adaptiveness. This also makes the whole model an online-learning model by an updated spatial model that learns the spatial distance for every frame.

The covariance matrix for this edge potential is selected as below: 

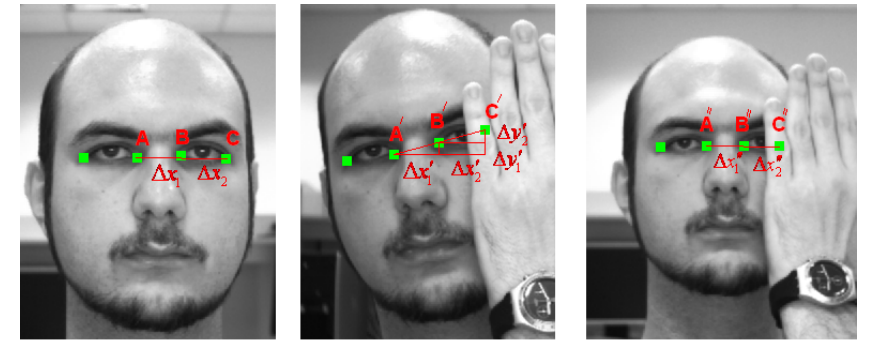

$$
\frac{\Delta x_{1}}{\Delta x_{2}}=\frac{\Delta x_{1}^{\prime}}{\Delta x_{2}^{\prime}}=\frac{\Delta y_{1}^{\prime}}{\Delta y_{2}^{\prime}}=\frac{\Delta x_{1}^{\prime \prime}}{\Delta x_{2}^{\prime \prime}}
$$

Figure 4. Adaptation of Affine-Ratio

$$
\Sigma=\left(\begin{array}{cccc}
\sigma_{x}^{\prime 2} & 0 & 0 & 0 \\
0 & \sigma_{y}^{\prime 2} & 0 & 0 \\
0 & 0 & \sigma_{u}^{\prime 2} & 0 \\
0 & 0 & 0 & \sigma_{v}^{\prime 2}
\end{array}\right)
$$

\subsection{Observation Model}

The extraction of the observations from video sequences is explained in section 2.1 .

The observation model makes the connection between the hidden random variable $x_{s}$ and the noisy local observation variable $y_{s}$. The model is as follows:

$$
y_{t}=C \cdot x_{t}+v \quad v \backsim N(0, R)
$$

Here, $C$ is the observation matrix and $R$ is the covariance matrix of the noise which is a normal distribution with zeromean. These are selected as follows:

$$
C=\left(\begin{array}{cccc}
1 & 0 & 0 & 0 \\
0 & 1 & 0 & 0
\end{array}\right) R=\left(\begin{array}{cc}
\sigma_{x}^{\prime \prime 2} & 0 \\
0 & \sigma_{y}^{\prime \prime 2}
\end{array}\right)
$$

As a result, the relation between $x_{s}$ and $y_{s}$ is a normal distribution and it is defined as follows:

$$
\psi_{s}\left(x_{s}, y_{s}\right)=N\left(C \cdot x_{s}, R\right)=p\left(y_{s} \mid x_{s}\right)
$$

\subsection{Loopy Belief Propagation Algorithm}

In many computer vision and image processing applications, the main target is to find the conditional density function $p\left(x_{s} \mid y\right)$. For graphs which are acyclic or tree-structured, the desired conditional distributions can be directly calculated by a local message-passing algorithm known as belief propagation (BP). In chain-structured graphs, this algorithm is equal to Kalman or Particle filtering. For cyclic graphs, Pearl [14] showed that belief propagation produces excellent empirical results in many cases. The algorithm is as follows: Each node $t \in V$ calculates a message $m_{t, s}\left(x_{s}\right)$ to be sent to each neighboring node

$$
\begin{aligned}
& s \in N(t): \\
& \alpha \int_{x_{t}} \psi_{s, t}\left(x_{s}, x_{t}\right) \psi_{t}\left(x_{t}, y_{t}\right) \times \prod_{u \in N(t) \backslash s} m_{u, t}\left(x_{s}\right) d x_{t}
\end{aligned}
$$

Each node combines these messages and its own observation and produces its own conditional density function :

$$
p\left(x_{s} \mid y\right)=\alpha \psi_{s}\left(x_{s}, y_{s}\right) \prod_{t \in N(s)} m_{t, s}\left(x_{s}\right)
$$

Since the relations in the model are selected as Gaussian, the two steps of the algorithm shown above simplify to updating means and covariances. For this reason, it works faster than non-parametric methods. Since the hidden random vector consists of the $\mathrm{x}, \mathrm{y}$ axis coordinates and velocities, the mean values of the normal distributions are the estimation of these values. Each update step is done using the current and the previous data, as a result the algorithm used becomes a filtering algorithm. For the update equations please see [15].

\section{Experimental Results}

The performance of the proposed work is shown by using the data recorded in a laboratory environment. The data consist of head movements, $x-y-z$ translation and rotation, and external occlusion.

For simplicity, only four eye corner points are tracked. The covariance matrices in the temporal, spatial and observation model are selected suitably according to the videos. The results of the proposed work are shown in Figure 6-b and 7-b. For comparison, the results of an algorithm that exploits only the temporal relations [7] are shown in Figure 6-a and 7-a. Green dots in the result images are the estimated point locations obtained using the observation data up to now.

As shown in Figure 6-b and 7-b, tracking of feature points is successfully done in the cases of arbitrary head movements and external occlusion. On the other hand, as seen in Figure 6-a and 7-a, the method that only use the temporal relation cannot track well and drifts occur and unreasonable tracking results.

The occlusion sequence is recorded by occluding a part of the face by hand. In this case the proposed occlusion detector, which simply thresholds the Gabor similarity values, detects the occlusion and the data term is closed considering the data are useless. The output of the similarity values for this video sequence is shown is Figure 5. The same occlusion detector is used both for the proposed method and the method in [7].

\section{Conclusion and Future Work}

In this paper a robust feature point tracker for applications, such human-computer interaction (HCI), facial ex- 


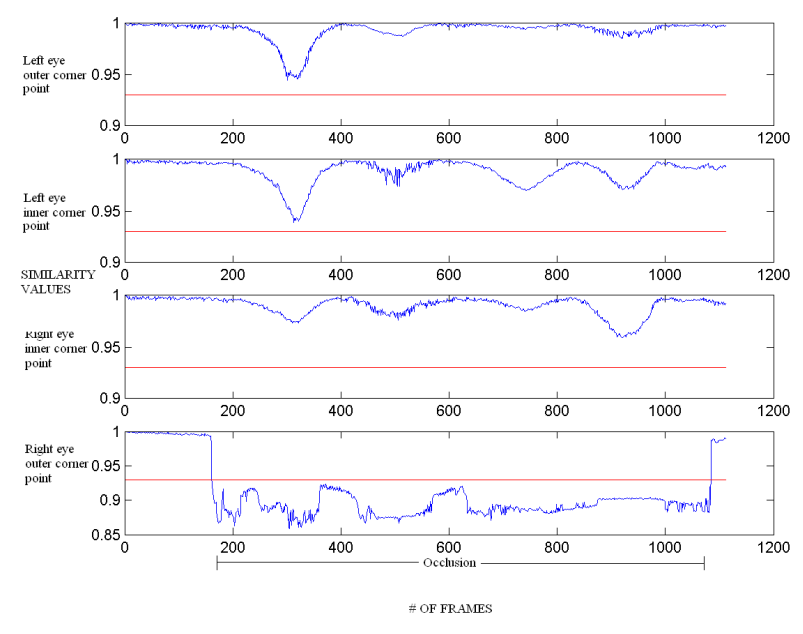

Figure 5. Similarity values of the four feature points for the video sequence in Figure 6 and 7

pression analysis etc., is developed. The significant advantage of the algorithm is the incorporation of the temporal and spatial information. So if a point disappears from the scene due to an occlusion, the information from the neighboring points will allow the tracking of the lost point to continue successfully. The spatial connection also provides a whole facial model to bind the feature points with each other and make them move altogether. To automatically detect occlusion an occlusion detector based on similarity values is proposed. Another advantage of the method is the computational efficiency. The parametric assumptions make computations simpler with respect to non-parametric techniques.

For future work, the model will be improved to track all facial feature points as an input to a facial expression analysis system .

\section{Acknowledgements}

This work was partially supported by the European Commission under Grants FP6-2004-ACC-SSA-2 (SPICE) and MIRG-CT-2006-041919, the Turkish State Planning Organization under the DRIVE-SAFE project, the New Energy and Industrial Technology Development Organization (NEDO) of Japan under the project "International Research Coordination of Driving Behavior Signal Processing based on Large Scale Real World Database”.

\section{References}

[1] Chetverikov D and Verestoy J, "Tracking feature points: a new algorithm," in Proceedings of the international conference on pattern recognition, 1998, p. 14361438.
[2] Rangarajan K and Shah M, "Establishing motion correspondence," CVGIP: Image Understanding, vol. 54, pp. 56-73, 1991.

[3] Bretzner L and Lindeberg T, "Feature tracking with automatic selection of spatial scales," Computer Vision Image Understanding, vol. 71, no. 3, pp. 385392, 1998.

[4] Shapiro L, Wang H, and Brady J, "A matching and tracking strategy for independently-moving, non-rigid objects," in Proceedings of the 3rd British machine vision conference, 1992, pp. 306-315.

[5] Meyer F and Bouthemy P, "Region-based tracking using affine motion models in long image sequences," Computer Vision Image Understanding, vol. 60, pp. 119140, 1994.

[6] Zheng Q and Chellappa R, "Automatic feature point extraction and tracking in image sequences for arbitrary camera motion," International Journal of Computer Vision, vol. 15, pp. 3176, 1995.

[7] $\mathrm{Gu} \mathrm{H}$ and Ji Q, "Information extraction from image sequences of real-world facial expressions," Mach. Vis. Appl., vol. 16, no. 2, pp. 105-115, 2005.

[8] Ji Q and Yang X, "Real-time eye, gaze, and face pose tracking for monitoring driver vigilance," Real-Time Imaging, vol. 8, no. 5, pp. 357-377, 2002.

[9] Cootes T F, Edwards G J, and Taylor C J, "Active appearance models," in Proceedings of the European conference on computer vision, 1998, p. 484498.

[10] Xiao J, Baker S, Matthews I, and Kanade T, "Realtime combined $2 \mathrm{~d}+3 \mathrm{~d}$ active appearance models," CVPR, vol. 2, pp. 535-542, 2004.

[11] Wan K, Lam K, and Chong N, "An accurate active shape model for facial feature extraction," Pattern Recognition Letters, vol. 26, no. 15, pp. 2409-2423, 2005.

[12] Su C and Huang L, "Spatio-temporal graphicalmodel-based multiple facial feature tracking," EURASIP Journal on Applied Signal Processing, vol. 13, pp. 2091-2100, 2005.

[13] David S. Bolme, "Elastic bunch graph matching," M.S. thesis, Colorado State University, 2003.

[14] J. Pearl, Probabilistic Reasoning in Intelligent Systems, Morgan Kaufman, San Mateo, 1988.

[15] E. Sudderth, "Embedded trees: Estimation of gaussian processes on graphs with cycles," M.S. thesis, MIT, 2002. 

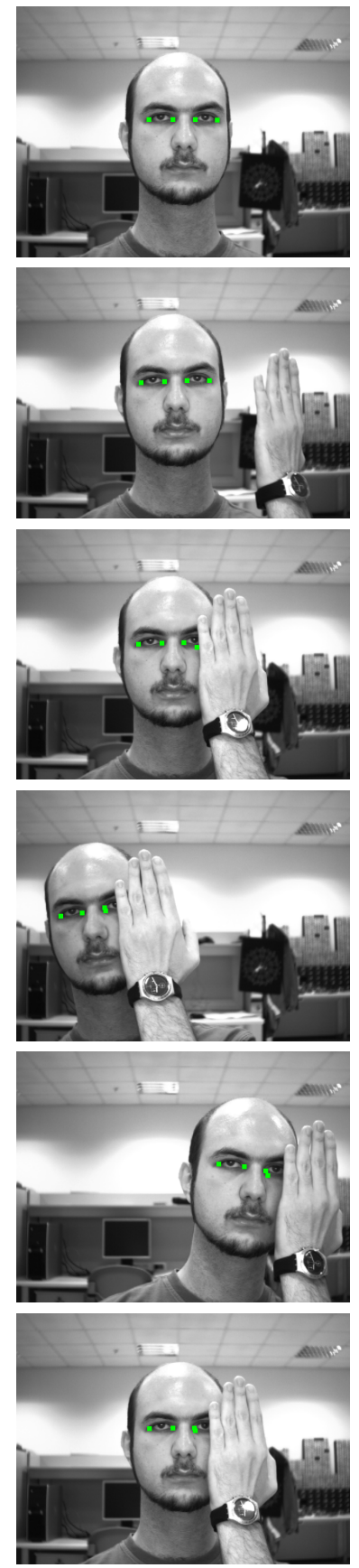

(a)
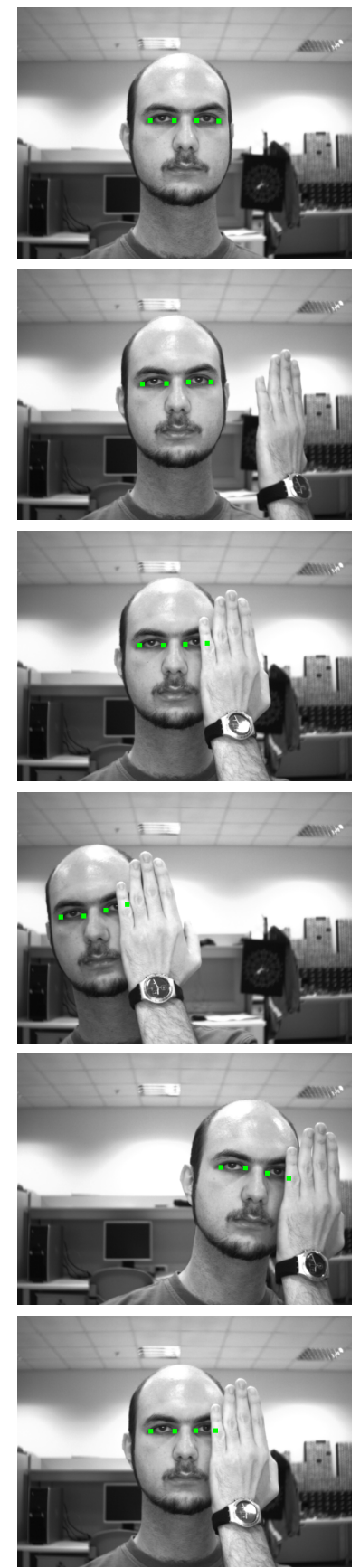

(b)

Figure 6. Tracking results of (a) the method in [7] (b) the proposed method for a head rotation sequence
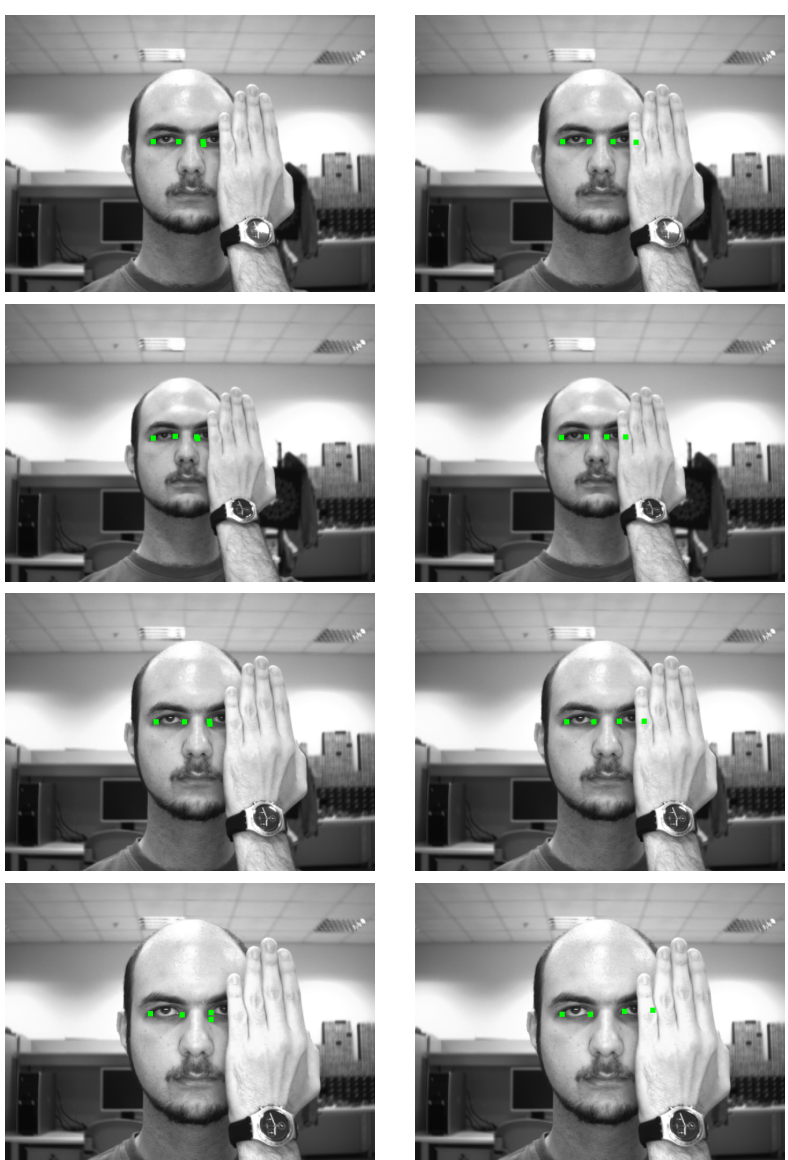

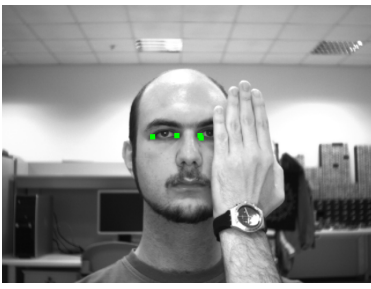

(a)

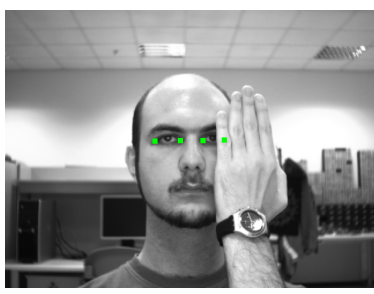

(b)
Figure 7. Tracking results of (a) the method in [7] (b) the proposed method for a head z-translation sequence 